\title{
Effects of Vibration Exercise on Muscle Performance and Mobility in an Older Population
}

\author{
Sven Rees, Aron Murphy, and Mark Watsford
}

\begin{abstract}
This study was designed to investigate the effects of vibration on muscle performance and mobility in a healthy, untrained, older population. Forty-three participants (23 men, 20 women, 66-85 y old) performed tests of sit-to-stand (STS), 5- and 10-m fast walk, timed up-and-go test, stair mobility, and strength. Participants were randomly assigned to a vibration group, an exercise-withoutvibration group, or a control group. Training consisted of 3 sessions/wk for $2 \mathrm{mo}$. After training, the vibration and exercise groups showed improved STS (12.4\%, $10.2 \%), 5$-m fast walk $(3.0 \%, 3.7 \%)$, and knee-extension strength $(8.1 \%, 7.2 \%)$ compared with the control $(p<0.05)$. Even though vibration training improved lower limb strength, it did not appear to have a facilitatory effect on functionalperformance tasks compared with the exercise-without-vibration group. Comparable mobility and performance changes between the experimental groups suggest that improvements are linked with greater knee-extension strength and largely attributed to the unloaded squats performed by both exercise groups.
\end{abstract}

Key Words: whole-body vibration, elderly, functional performance, strength

Aging is accompanied by sarcopenia, a decrease in muscle mass (Evans, 1995). The loss in muscle mass is directly linked to a decline in muscle strength and power. This decline can affect many aspects of physical function such as walking and rising from a chair (Brown, Sinacore, \& Host, 1995). In addition, muscle weakness and reduced ability to produce rapid force are considered two of the most common risk factors for increased risk of falls and loss of functional independence (Taaffe \& Marcus, 2000; Tinetti, Speechley, \& Ginter, 1988). To date, numerous studies have demonstrated that resistance training can increase muscle mass, strength, and power along with physical function (Fiatarone et al., 1990; Lamoureux, Sparrow, Murphy, $\&$ Newton, 2003). Accordingly, progressive resistance-training interventions are consistently prescribed to counteract age-related sarcopenia. An alternative exercise stimulus, whole-body vibration (WBV), has recently emerged as a potential training intervention that could positively influence the muscular system in older adults, but research investigating WBV exercise in the older population is sparse.

The authors are with the School of Leisure, Sport and Tourism, University of Technology, Sydney, Lindfiled NSW, 2070 Australia. 
WBV exercise involves standing on a platform that oscillates at a particular frequency and amplitude. Vibrations stimulate the neuromuscular system, activating muscles through spinal reflexes. These rapid vibrations stimulate muscle spindles, activating 1a $\alpha$-motoneurons, which initiate muscle contractions (Bishop, 1974). Such a phenomenon is known as the tonic vibration reflex. Current findings suggest that WBV can positively affect muscle performance (Delecluse, Roelants, \& Verschueren, 2003; Roelants, Delecluse, \& Verschueren, 2004). Other studies, however, have reported equivocal results (de Ruiter, Van Raak, Schilperoort, Hollander, \& de Haan, 2003; Schlumberger, Salin, \& Schmidtbleicher, 2001). The diversity of vibration-training protocols employed makes comparing results extremely difficult. The effects of WBV appear to depend on amplitude, frequency, intensity, volume, and activity undertaken (Luo, McNamara, \& Moran, 2005). A review of the literature reveals a clear need for research into the effects of chronic vibration (Luo et al.), particularly its effects on older adults.

It is well known that aging adults experience deficits in muscle function, a critical factor affecting physical function, mobility, and balance. Mobility is an essential component in sustaining functional independence with aging. Because vibration exercise might be an alternative method to increase strength and power in older adults, its correlation with changes in mobility warrants further research. The purpose of this study was, therefore, to investigate the effects of 2 months of WBV on muscle performance and mobility in a healthy, older population. It was hypothesized that WBV training would result in greater lower limb performance than the same exercise without vibration. This study provides an insight into the effectiveness of WBV as a training intervention for reduced functional performance and mobility in an older population.

\section{Methods}

\section{Participants and Study Design}

The sample size for the current study was determined a priori using measures of effect size and based on a number of findings reported in relevant literature (Bruyere et al., 2005; Roelants et al., 2004; Verschueren et al., 2004). Power analysis revealed that a sample size of 10 in the training groups was required to achieve power of .8 and $\alpha=.05$. To allow for possible dropouts, 15 participants in each group represented a sufficient quantity to determine the results to be meaningful.

Forty-five older adults were recruited for the study, but 2 in the exercisewithout-vibration group withdrew 2 weeks into the study for reasons not related to the exercise program. Forty-three healthy, older volunteers (23 men and 20 women age $66-85$ years) participated in the study. Grouped by gender, participants were randomized into three groups: 15 to a WBV training group (VIB), 13 to an exercise-without-vibration group (EX), and 15 to a control group (CONT). All participants were involved in low-intensity exercise (walking) at least three times per week. Although considered active, participants were not involved in any structured exercise regimen and were therefore classified as untrained. The exclusion criteria from the study were age $<65$ years; prosthesis; any neurological, musculoskeletal, or other chronic disease; involvement in a resistance-training program; a fracture or bone injury; and any medication that could adversely affect the results of the 
study. Each participant gave informed written consent to participate and provided clearance from a medical practitioner. The study and its procedures were approved by the university human research ethics committee.

\section{Overview of the Study}

The study was designed to evaluate the effectiveness of WBV exercise on physical performance and mobility. This was done by integrating previous WBV protocols and incorporating a progressive overload. Both treatment groups, VIB and EX, engaged in the same 8-week exercise regimen except that the VIB group performed all exercises on a vibration platform. The program consisted of two 4-week blocks: static squats (Bosco, Colli, et al., 1999; Runge, Rehfeld, \& Resnicek, 2000) and dynamic squatting and calf raises (Bosco et al., 1998; Verschueren et al., 2004). Resistance was provided by the vibration stimulus and through body-weight exercises. Both treatment groups were required to employ the same specific body positions: standing with the heels just off the ground and extending the hips to a maximum of $170^{\circ}$ when performing dynamic squatting. This was primarily designed for the VIB group to maximize vibration dampening and minimize transfer to the upper body. Participants in the VIB and EX groups were informed that they could use the handlebars on the vibration platform if they required extra support, but they were asked to limit their use to maximize the effect of training.

All training and testing were performed onsite at the university's humanperformance laboratory. Every training and testing session was directly supervised by the chief investigator. As such, the experimental protocol was not blinded. Training frequency was three times per week with at least 1 day of rest between sessions. Adherence to the training program was high, with $99 \% \pm 1 \%$ completion for both the VIB and EX groups. The outcome measures were performed prior to (before randomization) and after the 2-month training program. At the beginning of each testing and training session, a 5-min walking warm-up was performed. All participants completed a familiarization session for each test before the study commenced.

\section{Performance Tests}

Sit-to-Stand Test. The sit-to-stand (STS) test measured the time taken to rise from a chair five times, as fast and as safely as possible (Runge et al., 2000). This test was administered on a chair without arms and a seat height of $43.2 \mathrm{~cm}$. The chair was fixed to the ground to avoid movement during the test. A standardized position was used for each subject that included being seated in the middle of the chair, back straight, arms folded across the chest, and feet approximately shoulder width apart. Subjects were informed by the researcher when to begin and end the test. If an incorrect STS was observed an additional trial was conducted. A total of three trials were performed with 2 min rest between trials. The mean of the best two trials was used for analysis. The total time was measured using pressure pads fixed to the seat, with results recorded and calculated on a computer after each trial. Interday reliability of the STS test was .96.

Timed-Up and Go-Test. The timed-up and go-test (TUG) involved a participant's standing up from a chair, walking $3 \mathrm{~m}$ as quickly and as safely as possible, crossing 
a line marked on the floor, turning around, and then walking back to sit back down in the chair (Bruyere et al., 2005). The participant was seated in a 43.2-cm-high chair without arms, with his or her back flush against the backrest. The time taken was calculated by pressure pads secured to the center of the seat. Turning time, the time to turn around at the 3-m mark, was measured by two timing light gates (Swift Performance Equipment, Goonellabah, NSW, Australia) placed at $2.5 \mathrm{~m}$. The researcher gave a verbal cue to begin the test. The times for three trials were recorded, with 2 min rest between trials. The mean of the best two trials was used for analysis. Interday reliabilities of the TUG test and turning time were .92 and .90 , respectively.

Fast Walk. This test required participants to walk as fast as possible over $10 \mathrm{~m}$. The time taken was calculated using timing gates positioned at 5-m and $10-\mathrm{m}$ intervals from the start line. The starting position involved feet placed together, 30 $\mathrm{cm}$ behind the first timing gate. Once they were ready, participants were allowed to commence the test at their own discretion. It was a requirement of the walking test that one foot be kept in contact with the ground at all times. Three fast-walk trials were performed, with a 2-min rest period between trials. The mean of the two best trials was used for analysis. Interday reliabilities of the 5-m and 10-m fast-walk test were .95 and .98 , respectively.

Stair Mobility Test. The stair mobility task involved participants climbing and descending five steps $(17.8 \mathrm{~cm}$ high and $27.9 \mathrm{~cm}$ deep) with a $1-\mathrm{m}$ landing in between. The test measured the time taken to complete this task twice. Participants were required to ascend and descend one stair at a time and avoid using the handrail. Stair mobility was assessed by a video camera $(50 \mathrm{~Hz})$ placed $2 \mathrm{~m}$ perpendicular to the stairs. The total time taken was calculated by multiplying the number of frames by $1 / 50 \mathrm{~Hz}(0.02 \mathrm{~s})$. Participants were asked to undertake the course as quickly and safely as possible. Before assessment, two practice trials were allowed. A total of three trials were conducted, with a 2-min rest period between efforts. Interday reliability of the stair mobility task was .98 .

Isokinetic Strength. Dynamic muscle strength of the hip, knee, and ankle flexors and extensors was determined using a Cybex II (Lumex, Inc., Ronkonkoma, NY, USA) isokinetic dynamometer. A standardized warm-up of four submaximal muscle contractions was performed before each isokinetic test velocity. The angular velocity for the hip and knee was $60 \%$, and the ankle joint was tested at $30 \%$ s. Tests were performed in the following order: knee, ankle, and hip. To improve clarity of the results and discussion, only the right leg was used for analysis. Isokinetic testing involved three cyclic (uninterrupted) maximal repetitions, performed twice. Between trials, a 1-min rest period was imposed. Participants were instructed before each trial to contract specific muscles as fast and as hard as possible. Verbal encouragement was provided during the test to help participants produce maximum efforts.

Isokinetic testing of the hip, knee, and ankle flexors and extensors involved standardized body positioning. For all isokinetic tests, participants were strapped securely at the waist and chest. Participants were instructed to fold their arms across their chest for each contraction. Two stoppers were positioned to control joint range of motion. 
To test hip strength, subjects lay supine. The tested leg was placed at $90^{\circ}$ flexion, with the opposite leg supported in an extended position. The dynamometer arm was secured $5 \mathrm{~cm}$ above the patella. Hip-flexor and -extensor strength was measured from a neutral $0^{\circ}$ position to $90^{\circ}$ flexion.

Knee strength was assessed in the Cybex chair with the back positioned at $100^{\circ}$. The knees extended past the edge of the chair, with the lateral femoral condyle of the tested leg aligned with the axis of rotation of the dynamometer. The dynamometer arm was secured $5 \mathrm{~cm}$ above the medial malleolus. Knee-flexor and -extensor strength was measured from $85^{\circ}$ to $10^{\circ}$ of flexion.

Ankle strength was measured with subjects lying prone. Their knees were fully extended and stabilized. The tested foot was fixed to the dynamometer footplate, with the ankle maintained at $10^{\circ}$ dorsiflexion. The lateral malleolus was aligned with the dynamometer axis of rotation. The tested leg was secured with a Velcro strap $5 \mathrm{~cm}$ below the patella. Ankle-flexor and -extensor strength was measured from $10^{\circ}$ dorsiflexion to $20^{\circ}$ plantar flexion.

The Cybex was calibrated before testing, using known masses placed on the lever arm. A gravity correction factor (additional torque created by the mass of limb) was determined by measuring the mass of the limb through its range of motion before each test. The procedures for isokinetic testing were based on a review of literature outlined by Perrin (1993) and shown to be both valid and reliable.

Vibration Exercise. The VIB participants were exposed to vertical sinusoidal WBV using a Galileo Sport platform (Novotec, Pforzheim, Germany). The frequency for this study was set at $26 \mathrm{~Hz}$ (Bosco et al., 1998; Bruyere et al., 2005), with peak-to-peak amplitude ranging from 5 to $8 \mathrm{~mm}$. The WBV protocol was designed to facilitate muscle and power development, based on an extensive review of literature. The training intensity and volume were progressively increased according to the overload principle. Training volume systematically increased with longer vibration sessions. Training intensity was increased by progressively raising the amplitude and incorporating dynamic lower limb exercises. This progressive overload is displayed in Table 1.

\section{Table 1 Training Volume and Training Intensity of the Whole-Body- Vibration (WBV) Program}

\begin{tabular}{ccccccc}
\hline & $\begin{array}{c}\text { WBV } \\
\text { duration } \\
(\mathbf{s})\end{array}$ & Rest (s) & $\begin{array}{c}\text { Amplitude } \\
(\mathbf{m m})\end{array}$ & $\begin{array}{c}\text { Frequency } \\
\mathbf{( H z )}\end{array}$ & Exercise & $\begin{array}{c}\text { Total exercise } \\
\text { duration (min) }\end{array}$ \\
\hline Week 1 & $6 \times 45$ & $5 \times 45$ & 5 & 26 & SWBK & 4.5 \\
Week 2 & $6 \times 50$ & $5 \times 50$ & 5 & 26 & SWBK & 5 \\
Week 3 & $6 \times 55$ & $5 \times 55$ & 6 & 26 & SWBK & 5.5 \\
Week 4 & $6 \times 60$ & $5 \times 60$ & 6 & 26 & SWBK & 6 \\
Week 5 & $6 \times 65$ & $5 \times 65$ & 7 & 26 & DLLE & 6.5 \\
Week 6 & $6 \times 70$ & $5 \times 70$ & 7 & 26 & DLLE & 7 \\
Week 7 & $6 \times 75$ & $5 \times 75$ & 8 & 26 & DLLE & 7.5 \\
Week 8 & $6 \times 80$ & $5 \times 80$ & 8 & 26 & DLLE & 8 \\
\hline
\end{tabular}

Note. SWBK = standing with bent knees; DLLE = dynamic lower limb exercises. 
The Borg 15-category scale for rating of perceived exertion (RPE) was used to monitor training intensity. Participants were asked to report their RPE scores after the fourth exercise set during every training session.

\section{Statistical Analyses}

Descriptive statistics are reported as $M \pm S D$. Before training, a one-way analysis of variance (ANOVA) was used to determine whether there were differences between groups. On completion of the training period, the data were examined for normality of distribution, with between-groups effects determined by a one-way ANCOVA, using the pretest values as the covariates. An ANCOVA was used to account for the variation in pretest values within each group. An ANCOVA is an appropriate statistical procedure for comparing groups that exhibit large variations in a dependent variable (Vincent, 2005). It is common to use an ANCOVA for variables such as strength, which might experience disproportional effects in the experiment, therefore adjusting the posttest scores to reflect the effects of the treatment (Vincent). Effect sizes are reported as partial eta squared $\left(\eta_{\mathrm{p}}{ }^{2}\right)$. For all between-groups procedures, significance was accepted at the alpha level of .05.

\section{Results}

There were no significant differences between groups for any variable at the pretests (Tables 2-4). The time effects of training for the variables are displayed in Tables 3 and 4.

\section{Rating of Perceived Exertion}

There were no significant differences in the reported RPE between the VIB and EX groups for all but one training occasion. The only significant difference in RPE occurred in Session $13\left(p=.04, \eta_{\mathrm{p}}{ }^{2}=.150\right.$; Figure 1). This corresponded with the change in training program from static to dynamic exercises. The difference in reported RPE between groups returned to a nonsignificant value after Training Session 14.

\section{Table 2 Basic Characteristics of the Vibration, Exercise, and} Control Groups, $M \pm \mathrm{SD}$

\begin{tabular}{lccc}
\hline & Vibration, $\boldsymbol{n}=\mathbf{1 5}$ & Exercise, $\boldsymbol{n}=\mathbf{1 3}$ & Control, $\boldsymbol{n}=\mathbf{1 5}$ \\
\hline Age (years) & $74.3 \pm 5.0$ & $73.1 \pm 4.1$ & $73.1 \pm 4.6$ \\
Height $(\mathrm{cm})$ & $167.5 \pm 10.9$ & $168.7 \pm 10.6$ & $168.3 \pm 10.3$ \\
Body mass $(\mathrm{kg})$ & & & \\
$\quad$ pre & $75.3 \pm 12.6$ & $75.9 \pm 8.9$ & $72.5 \pm 11.8$ \\
$\quad$ post & $75.3 \pm 13.3$ & $75.7 \pm 8.5$ & $72.4 \pm 12.0$ \\
\hline
\end{tabular}


Table 3 Muscle Performance and Mobility Results for the Vibration, Exercise, and Control Groups, $M \pm S D$

\begin{tabular}{|c|c|c|c|c|}
\hline & Time & Vibration, $n=15$ & Exercise, $n=13$ & Control, $n=15$ \\
\hline \multirow[t]{2}{*}{ TUG time (s) } & pre & $5.19 \pm 0.39$ & $5.29 \pm 0.66$ & $5.09 \pm 0.64$ \\
\hline & post & $4.89 \pm 0.29 *$ & $5.04 \pm 0.47$ & $5.04 \pm 0.66$ \\
\hline \multirow[t]{2}{*}{ TUG turning time (s) } & pre & $1.37 \pm 0.15$ & $1.39 \pm 0.19$ & $1.36 \pm 0.18$ \\
\hline & post & $1.33 \pm 0.12$ & $1.35 \pm 0.17$ & $1.33 \pm 0.23$ \\
\hline \multirow[t]{2}{*}{ Fast-walk 5-m time (s) } & pre & $2.37 \pm 0.19$ & $2.46 \pm 0.23$ & $2.38 \pm 0.23$ \\
\hline & post & $2.30 \pm 0.15^{*}$ & $2.37 \pm 0.22 *$ & $2.38 \pm 0.23$ \\
\hline \multirow{2}{*}{$\begin{array}{l}\text { Fast-walk 10-m time } \\
\text { (s) }\end{array}$} & pre & $4.52 \pm 0.45$ & $4.60 \pm 0.56$ & $4.49 \pm 0.64$ \\
\hline & post & $4.30 \pm 0.40^{*}$ & $4.42 \pm 0.54$ & $4.45 \pm 0.59$ \\
\hline \multirow[t]{2}{*}{ STS time (s) } & pre & $9.91 \pm 0.80$ & $9.95 \pm 0.83$ & $9.82 \pm 1.15$ \\
\hline & post & $8.69 \pm 0.79 *$ & $8.93 \pm 0.99 *$ & $9.73 \pm 1.21$ \\
\hline \multirow[t]{2}{*}{ Stair mobility time (s) } & pre & $8.09 \pm 0.91$ & $8.37 \pm 1.44$ & $8.18 \pm 1.52$ \\
\hline & post & $7.91 \pm 0.84$ & $8.21 \pm 1.37$ & $8.11 \pm 1.65$ \\
\hline
\end{tabular}

Note. TUG = timed up-and-go; STS = sit-to-stand.

*Significantly greater change compared with CONT group $(p<.05)$.

Table 4 Isokinetic Strength Results for the Vibration, Exercise, and Control Groups, N/m, $M \pm$ SD

\begin{tabular}{llccc}
\hline & Time & $\begin{array}{c}\text { Vibration \%BW, } \\
\boldsymbol{n}=\mathbf{1 5}\end{array}$ & $\begin{array}{c}\text { Exercise \%BW, } \\
\boldsymbol{n}=13\end{array}$ & $\begin{array}{c}\text { Control \%BW, } \\
\boldsymbol{n}=15\end{array}$ \\
\hline $\begin{array}{l}\text { Right knee-flexion } \\
\text { torque }\end{array}$ & pre & $102.4 \pm 27.1$ & $109.1 \pm 35.3$ & $106.7 \pm 32.4$ \\
& post & $113.6 \pm 29.4$ & $116.1 \pm 36.3$ & $109.0 \pm 32.4$ \\
$\begin{array}{l}\text { Right knee-extension } \\
\text { torque }\end{array}$ & pre & $164.5 \pm 35.4$ & $167.4 \pm 44.4$ & $165.8 \pm 37.1$ \\
& post & $177.8 \pm 34.4^{*}$ & $179.5 \pm 41.8^{*}$ & $163.4 \pm 38.3$ \\
$\begin{array}{l}\text { Right ankle- } \\
\text { dorsiflexion torque }\end{array}$ & pre & $35.5 \pm 7.8$ & $37.1 \pm 10.6$ & $37.3 \pm 6.4$ \\
& post & $36.1 \pm 7.0$ & $37.4 \pm 10.7$ & $36.8 \pm 6.1$ \\
$\begin{array}{l}\text { Right ankle-plantar- } \\
\text { flexion torque }\end{array}$ & pre & $101.6 \pm 23.2$ & $104.8 \pm 31.1$ & $108.1 \pm 16.7$ \\
& post & $120.4 \pm 24.4^{* \dagger}$ & $110.2 \pm 31.4$ & $109.2 \pm 18.1$ \\
$\begin{array}{l}\text { Right hip-flexion } \\
\text { torque }\end{array}$ & pre & $139.3 \pm 28.9$ & $136.5 \pm 48.1$ & $140.3 \pm 28.8$ \\
& post & $143.8 \pm 29.2$ & $141.2 \pm 44.3$ & $140.6 \pm 27.2$ \\
$\begin{array}{l}\text { Right hip-extension } \\
\text { torque }\end{array}$ & pre & $139.3 \pm 28.9$ & $136.5 \pm 48.1$ & $140.3 \pm 28.8$ \\
& post & $143.8 \pm 29.2$ & $141.2 \pm 44.3$ & $140.6 \pm 27.2$ \\
\hline
\end{tabular}

Note. $\mathrm{BW}=$ body weight.

$*$ Significantly greater change than in CONT group $(p<.05)$. $†$ Significantly greater change than in EX group $(p<.05)$. 


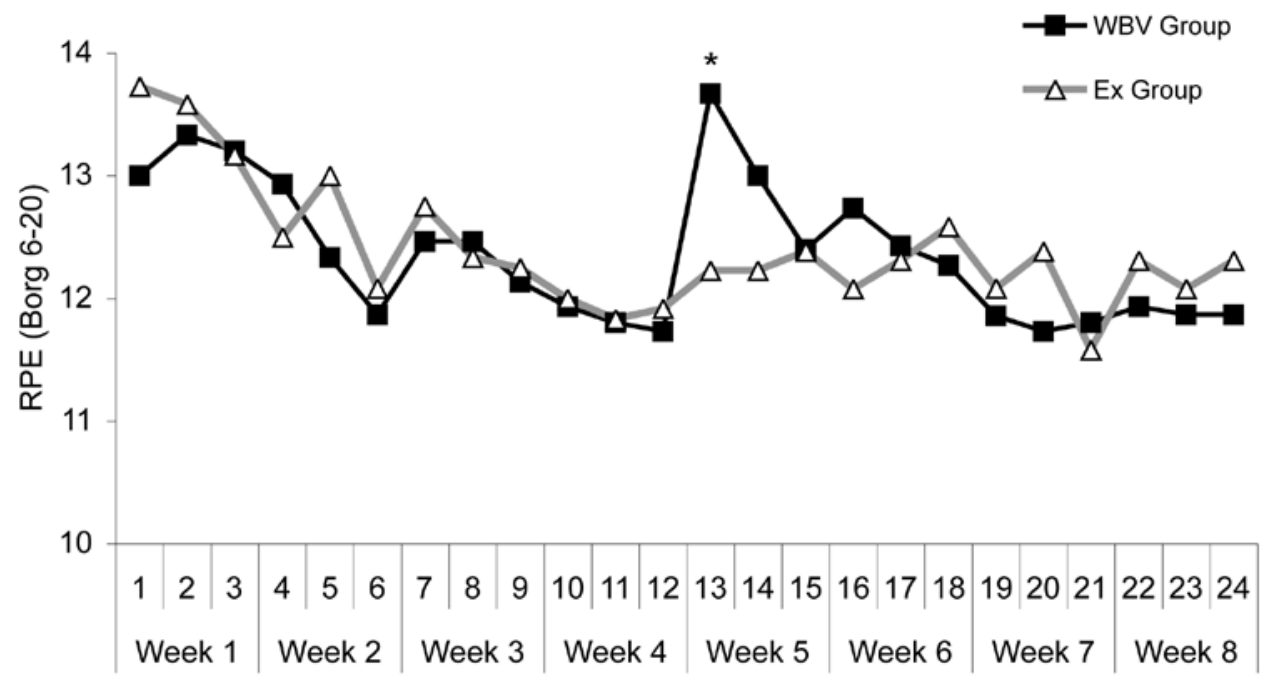

Figure 1 - Mean ratings of perceived exertion (RPE) for whole-body vibration (WBV) and exercise (Ex) training. *Significantly greater change than in the Ex group $(p<.05)$.

\section{TUG Test}

The TUG test displayed significant differences for the amount of change in the VIB group compared with the CONT group from pre- to posttest $\left(p=.040, \eta_{\mathrm{p}}{ }^{2}=.153\right)$. There were no significant differences between the VIB and EX or EX and CONT groups for the TUG test. No differences were evident for turning time.

\section{Fast Walk}

When comparing pre- and posttest values, a significant difference was evident between the VIB and EX groups versus the CONT group over $5 \mathrm{~m}$ (VIB vs. CONT $5 \mathrm{~m}: p=.044, \eta_{\mathrm{p}}{ }^{2}=.184 ;$ EX vs. CONT $\left.5 \mathrm{~m}: p=.045, \eta_{\mathrm{p}}{ }^{2}=.184\right)$ and VIB versus CONT group over $10 \mathrm{~m}\left(p=.008, \eta_{\mathrm{p}}{ }^{2}=.218\right)$. A trend was evident for $10-\mathrm{m}$ fast walk from pre- to posttraining between EX and CONT $\left(p=.089, \eta_{\mathrm{p}}{ }^{2}=.218\right)$. There were no main effects between the VIB and EX groups from pre- to posttesting.

\section{STS Performance}

The VIB and EX groups displayed significant differences compared with the CONT group for the magnitude of STS improvement from the pre- to posttesting occasion $\left(p<.001, \eta_{\mathrm{p}}^{2}=.553\right)$. There were no differences in STS performance between the VIB and EX groups after the 2-month training period.

\section{Stair Mobility}

There were no significant differences between groups for the stair mobility test. The results for stair mobility for each group are displayed in Table 3. 


\section{Isokinetic Strength}

Hip-strength results pre- to posttesting revealed no significant difference or trends between groups. At the completion of 2 months training, a nonsignificant trend was evident for changes in right knee-flexion torque between the VIB and CONT groups $\left(p=.086, \eta_{\mathrm{p}}{ }^{2}=.117\right)$. After the 2 months of training there were significant knee-extension-torque changes for the VIB and EX groups compared with the CONT group $\left(p<.001, \eta_{\mathrm{p}}{ }^{2}=.315\right)$.

The VIB group displayed significant improvements after 2 months of training in ankle-plantar-flexion strength compared with EX and CONT pretest values ( $p<$ $\left..001, \eta_{\mathrm{p}}{ }^{2}=.431\right)$. There were no main effects for ankle-dorsiflexion strength pre- to posttesting. The isokinetic-strength results are displayed in Table 4.

\section{Discussion}

The primary purpose of this study was to examine the results of 2 months of WBV and exercise without vibration training on a battery of muscle-performance and mobility measures in a healthy older population. The results showed that improvements in the TUG, fast walking, and STS performance after training were comparable between the VIB and EX groups. Although WBV training did not appear to have a facilitatory effect over the unloaded exercise for these specific performance measures, it appeared to improve the strength-training response in the triceps surae muscles.

The decline in muscle strength with aging is associated with decreased mobility and impaired performance of functional activities (Brown et al., 1995). The VIB group exhibited a significant facilitatory effect for plantar-flexion strength, $18.5 \%$, compared with $5.2 \%$ for the EX group. In comparison, there were no significant differences for knee-flexion and -extension strength gains between the VIB and EX groups. Because the flexor and extensor muscles of the knee and hip would influence mobility and performance measures to a greater degree than the ankle plantar flexors, the comparable improvements between the VIB and EX groups appears to reflect strength gains in these muscles. Although strength is considered a component of mobility and functional performance (Brown et al.), the facilitated gains in plantar-flexion strength for the VIB group did not transfer to greater improvements in mobility and functional performance than in the EX group.

An important component of exercise prescription for an older population is understanding the influence of specific muscles on mobility and functional performance. Previous research has shown hip extension as the major action responsible for gait characteristics in elderly men (Burnfield, Josephson, Powers, \& Rubenstein, 2000). In other research, knee-extension strength and power were found to be significantly associated with the performance of gait, STS, and stair climbing in an older population (Bean et al., 2002). As such, the prescription of strength training for functional movement should specifically target these muscle groups. Although a few studies have investigated the effects of WBV on knee strength in older adults (Roelants et al., 2004; Verschueren et al., 2004), no other authors have looked at its effect on the hip flexors and extensors. Given the similarities in knee- and hip-strength gains between the VIB and EX groups it is not surprising that we also observed comparable improvements in TUG, STS, and fast walking. The exercise protocol 
used in the current study might provide an explanation for the resultant performance changes. The WBV platform transmits a vibration wave to the lower body that is dissipated distal to proximally by the muscles. Because it is well documented that vibration can have a negative effect on human health (Seidel \& Heide, 1986), and tied in with previous reports stressing the importance of minimizing its transfer to the upper body (Mester, Spitzenfeil, Schwarzer, \& Seifriz, 1999), the current study employed specific body positions to maximize dampening in the lower extremities. Two body positions that were employed included a plantar-flexion contraction to ensure that the heels were slightly raised and that the knees and hips were never extended completely. Body position can significantly alter the transfer of vibration to the proximal body segments (Crewther, Cronin, \& Keogh, 2004). It is therefore plausible that incorporating various exercises or body positions that result in greater attenuation of vibration in the knee and hip muscles could facilitate strength gains and lead to improved functional performance. Future WBV interventions should include a protocol that can target muscles higher up on the leg.

In its simplest form, there are two components to WBV training: the vibration stimulus and the specific exercises performed on the platform. Although WBV training is reported to facilitate strength gains, it must be acknowledged that the unloaded exercises performed on the platform can also contribute to the improvements in strength, particularly in older adults. The results of a recent placebo-controlled study suggest that the major contribution to strength gains after WBV is the reflex muscle contractions it provokes (Delecluse et al., 2003). WBV provided a facilitatory effect on ankle plantar-flexion strength, indicating that this training stimulus affects the muscles proximal to the vibration stimulus. We did not find a facilitatory effect for the WBV training group on knee-extension strength similar to that reported by Delecluse et al. (2003), nor were there significantly larger gains in TUG, fast walking, STS, or stair locomotion. It must be noted that there were differences in methodology between the present study at that of Delecluse et al. (2003), for example, young versus older participants, 3 months training compared with 2 , and slight variations in vibration frequency and amplitude. A major difference between these studies appears to be the exercises undertaken. Additional exercises such as deep squat, wide-stance squat, one-legged squat, and lunge were employed in the research by Delecluse et al. (2003). These additional exercises would promote a greater intensity of training, potentially facilitating greater WBV strength gains (Ronnestad, 2004). Second, by incorporating different body positions and exercises, the degree of muscle activation induced by WBV would vary in the muscles of the lower body. Although there has been research conducted on the level of muscle activity induced by WBV in various muscles, no study has investigated the long-term effects of strength changes after WBV using two different body positions or exercise regimens.

WBV training increases muscle activity for a number of muscle groups of the lower body at the same time. The muscles closer to the platform, however, show a higher vibration effect (Roelants, Verschueren, Delecluse, Levin, \& Stijnen, 2006). Because WBV provides a strong stimulus to the neuromuscular system, increased plantar-flexion strength might be connected with neural potentiation, similar to traditional strength-training adaptations. This notion is in line with the practice of incorporating WBV training to stimulate the neuromuscular system and attain gains in neuromuscular performance (Bosco, Cardinale, \& Tsarpela, 1999; Luo 
et al., 2005). It must be noted that measuring mechanisms and adaptations after WBV was outside the scope of this study. There is still a lack of knowledge about the effects of WBV on the neuromuscular system (Roelants et al., 2006). There is a need for research into neuromuscular adaptations after WBV exercise.

Only a handful of studies have been published examining the effects of WBV in older adults (Bautmans, Van Hees, Lemper, \& Mets, 2005; Bruyere et al., 2005; Roelants et al., 2004; Runge et al., 2000; Russo et al., 2003; Verschueren et al., 2004). Similar to the current study, Bautmans et al. examined WBV compared with the same exercise without vibration, demonstrating a 3-s improvement in TUG time for the WBV group. When other results from Bautmans et al. are analyzed, however, an overall picture of WBV performance enhancement is less clear. In agreement with results of the current study, Bautmans et al. also reported improvements in isokinetic leg-extension strength for both the WBV group and exercise-withoutvibration group, with no significant differences between the groups. It could be argued that older participants' characteristics could explain the similar performance gains between the VIB and EX groups in the current study. Because the older adults exhibit reduced strength in comparison with the young population, they would appear to have greater potential for performance enhancement. These lower initial strength scores might explain the comparable knee-strength gains in both the VIB and the EX group. It must be acknowledged, however, that WBV did facilitate superior plantar-flexion strength in the current study. The $18.5 \%$ improvement in plantar-flexion strength (Table 4) after the training period revealed that the older participants in the current study approached values similar to those reported in a healthy, younger population (mean age 23.4 years, range 19-29). Maximum ankle torques at the pretest were similar to those in an age-matched group (Thelen, Schultz, Alexander, \& Ashton-Miller, 1996), but at the posttest occasion, these values were within $1 S D$ of the young population's mean. This indicates a clinically significant improvement in this measure.

Improved ankle-plantar-flexion strength after WBV training can be considered an important adaptation for an older population. This muscle group exhibits a significant decline in strength with the aging process (Simoneau, Martin, \& Van Hoecke, 2005). In older adults, distal muscle groups are also considered more susceptible to the aging-related motor-unit loss then more proximal muscles (Galea, 1996). Because ankle-plantar-flexion strength plays an important role in mobility and balance in the older population (Kerrigan, Todd, Della Croce, Lipsitz, \& Collins, 1998; Onambele, Narici, \& Maganaris, 2006; Suzuki, Bean, \& Fielding, 2001), WBV might serve as a supplement to other training methods in mediating functional decline. In the current study, however, when compared with the EX group, the increase in plantar-flexion strength with WBV training did not reveal significant performance enhancements in the tests conducted. WBV might have the potential to improve mobility and functional performance in an older population, but more research is required to examine the relationship between increased plantar-flexion strength and various functional tasks.

There is a paucity of information on the effect of WBV on mobility and more complex skills. There have been reports that WBV training does not have an effect on shuttle-run (Torvinen et al., 2002b), sprinting speed (Delecluse, Roelants, Diels, Koninckx, \& Verschueren, 2005) or agility performance (Cochrane, Legg, \& Hooker, 2004). In contrast, other studies have reported that WBV positively 
influenced TUG (Schuhfried, Mittermaier, Jovanovic, Pieber, \& Paternostro-Sluga, 2005), Tinetti test (Bautmans et al., 2005; Bruyere et al., 2005), and balance (Torvinen et al., 2002a). These variations might be explained by variations in the complexity of the task or the fact that transfer to functional performance reflects the nature of the activity undertaken during WBV. Stimulating the muscles while performing an activity that is similar to the measured performance test should result in a greater transfer. STS performance closely reflects the squatting training undertaken and demonstrated large improvements in both training groups after 2 months of training (VIB: 12.4\%; EX: 10.2\%; CONT: 0.9\%). This is in agreement with Runge et al. (2000), who observed an $18 \%$ increase with 2 months of static WBV training compared with a nonexercising control. The improvements in the various mobility and muscle-performance measures in the current study might indicate a transfer effect, mediated through the specific motor pattern employed during training (static and dynamic squats). Although STS performance closely mirrors the training interventions, smaller changes in turning time or stair climbing appear to reflect training specificity, where there is less transfer the more complex the task. If WBV training is to transfer to improved functional performance it should employ protocols that reflect the nature of that activity. Because mobility is an essential component of functional independence in the elderly, future WBV protocols should be varied and based on the specificity principle.

Resistance-training programs can improve strength and power in older adults (Fiatarone et al., 1990). Because both VIB and EX participants reported that the unloaded static and dynamic exercises were somewhat hard (Figure 1), it is reasonable to attribute some of the strength changes after training to the activity performed. On one occasion, a significant difference in RPE was evident, yet this returned to a nonsignificant difference at the next training session. The higher RPE in the VIB group corresponded to the incorporation of dynamic squats and calf raises, described by participants as entailing extra difficulty in maintaining balance. Similarities in RPE have also been found after squatting with and without exhaustive WBV (Rittweger, Mutschelknauss, \& Felsenberg, 2003). The effects of WBV are influenced by numerous factors including amplitude, frequency, exercises performed, intensity, and volume. To date, every WBV review article reiterates that an optimal WBV protocol is still unclear (Issurin, 2005; Jordan, Norris, Smith, \& Herzog, 2005; Luo et al., 2005). There is a distinct need for future studies to address this paucity of information. The outcomes of such work will enable more effective prescription and a greater understanding of the efficacy of WBV, particularly its use as a rehabilitation tool to combat functional decline in older adults.

Over the last couple of years, WBV has become more accessible, with platforms now located at a number of gyms, hospitals, and nursing homes. In addition, a number of companies have developed units for the home market. Because WBV training can be conducted in one's home, it might serve as an alternative exercise intervention for older adults who are not attracted to or are unable to perform traditional resistance training. The general public, however, knows relatively little about vibration platforms. The efficacy of WBV as reported in the literature is based on a relatively small time commitment required, its ease of use (standing or squatting with knees flexed on the platform), low aerobic demands, mild effects on blood pressure (Rittweger, Beller, \& Felsenberg, 2000), its use as a therapy 
for osteoporosis, improved neuromuscular efficiency, and its potential for large performance enhancements (Bosco, Colli, et al., 1999). These positive effects highlight the need for more knowledge of the potential use of WBV exercise in an older population.

\section{Conclusion}

The current study found that WBV training did have a facilitatory effect on ankle plantar-flexion strength. WBV training did not increase TUG, fast-walking, or STS performance to a significantly greater extent than in the exercise-without-vibration group. The gains in strength for both the VIB and EX group can account for the observed improvements in performance measures compared with the CONT group. The exercise protocols undertaken appear to account for reported knee-strength gains and the transfer to dynamic muscle performance and mobility measures. WBV might have the potential to facilitate mobility in a healthy older population. More research needs to be conducted, however, on the various exercises and vibration protocols that transfer to improved strength in key muscles such as the knee and hip.

\section{References}

Bautmans, I., Van Hees, E., Lemper, J.C., \& Mets, T. (2005). The feasibility of whole body vibration in institutionalised elderly persons and its influence on muscle performance, balance and mobility: A randomised controlled trial. BMC Geriatrics, 5(1), 17. Retrieved August 17, 2007, from www.biomedcentral.com/content/pdf/1471-2318-5-17.pdf

Bean, J.F., Kiely, D.K., Herman, S., Leveille, S.G., Mizer, K., Frontera, W.R., et al. (2002). The relationship between leg power and physical performance in mobility-limited older people. Journal of the American Geriatrics Society, 50(3), 461-467.

Bishop, B. (1974). Vibration stimulation. Part 1. Neurophysiology of motor responses evoked by vibration stimulation. Physical Therapy, 54(12), 1273-1282.

Bosco, C., Cardinale, M., \& Tsarpela, O. (1999). Influence of vibration on mechanical power and electromyogram activity in human arm flexor muscles. European Journal of Applied Physiology and Occupational Physiology, 79(4), 306-311.

Bosco, C., Cardinale, M., Tsarpela, O., Colli, R., Tihanyi, J., Duvillard, S., et al. (1998). The influence of whole body vibration on jumping performance. Biology in Sport, 15(3), 157-164.

Bosco, C., Colli, R., Introini, E., Cardinale, M., Tsarpela, O., Madella, A., et al. (1999). Adaptive responses of human skeletal muscle to vibration exposure. Clinical Physiology, 19(2), 183-187.

Brown, M., Sinacore, D.R., \& Host, H.H. (1995). The relationship of strength to function in the older adult. Journal of Gerontology A: Biological Sciences and Medical Sciences, 50, 55-59.

Bruyere, O., Wuidart, M.A., Di Palma, E., Gourlay, M., Ethgen, O., Richy, F., et al. (2005). Controlled whole body vibration to decrease fall risk and improve health-related quality of life of nursing home residents. Archives of Physical Medicine and Rehabilitation, 86(2), 303-307.

Burnfield, J.M., Josephson, K.R., Powers, C.M., \& Rubenstein, L.Z. (2000). The influence of lower extremity joint torque on gait characteristics in elderly men. Archives of Physical Medicine and Rehabilitation, 81(9), 1153-1157. 
Cochrane, D.J., Legg, S.J., \& Hooker, M.J. (2004). The short-term effect of whole-body vibration training on vertical jump, sprint, and agility performance. Journal of Strength and Conditioning Research, 18(4), 828-832.

Crewther, B., Cronin, J., \& Keogh, J. (2004). Gravitational forces and whole body vibration: Implications for prescription of vibratory stimulation. Physical Therapy in Sport, 5, 37-43.

Delecluse, C., Roelants, M., Diels, R., Koninckx, E., \& Verschueren, S. (2005). Effect of whole body vibration training on muscle strength and sprint performance in sprinttrained athletes. International Journal of Sports Medicine, 26(8), 662-668.

Delecluse, C., Roelants, M., \& Verschueren, S. (2003). Strength increase after whole-body vibration compared with resistance training. Medicine and Science in Sports and Exercise, 35(6), 1033-1041.

de Ruiter, C.J., Van Raak, S.M., Schilperoort, J.V., Hollander, A.P., \& de Haan, A. (2003). The effects of 11 weeks whole body vibration training on jump height, contractile properties and activation of human knee extensors. European Journal of Applied Physiology, 90(5-6), 595-600.

Evans, W.J. (1995). What is sarcopenia? Journals of Gerontology. Series A, Biological Sciences and Medical Sciences, 50A, 5-8.

Fiatarone, M.A., Marks, E.C., Ryan, N.D., Meredith, C.N., Lipsitz, L.A., \& Evans, W.J. (1990). High-intensity strength training in nonagenarians. Effects on skeletal muscle. Journal of the American Medical Association, 263(22), 3029-3034.

Galea, V. (1996). Changes in motor unit estimates with aging. Journal of Clinical Neurophysiology, 13(3), 253-260.

Issurin, V.B. (2005). Vibrations and their applications in sport. A review. Journal of Sports Medicine and Physical Fitness, 45(3), 324-336.

Jordan, M.J., Norris, S.R., Smith, D.J., \& Herzog, W. (2005). Vibration training: An overview of the area, training consequences, and future considerations. Journal of Strength and Conditioning Research, 19(2), 459-466.

Kerrigan, D.C., Todd, M.K., Della Croce, U., Lipsitz, L.A., \& Collins, J.J. (1998). Biomechanical gait alterations independent of speed in the healthy elderly: Evidence for specific limiting impairments. Archives of Physical Medicine and Rehabilitation, 79(3), 317-322.

Lamoureux, E., Sparrow, W.A., Murphy, A., \& Newton, R.U. (2003). The effects of improved strength on obstacle negotiation in community-living older adults. Gait and Posture, 17(3), 273-283.

Luo, J., McNamara, B., \& Moran, K. (2005). The use of vibration training to enhance muscle strength and power. Sports Medicine, 35(1), 23-41.

Mester, J., Spitzenfeil, P., Schwarzer, J., \& Seifriz, F. (1999). Biological reaction to vibration-Implications for sport. Journal of Science and Medicine in Sport, 2(3), 211226.

Onambele, G.L., Narici, M.V., \& Maganaris, C.N. (2006). Calf muscle-tendon properties and postural balance in old age. Journal of Applied Physiology, 100(6), 2048-2056.

Perrin, D. (1993). Isokinetic exercise and assessment. Champaign, IL: Human Kinetics.

Rittweger, J., Beller, G., \& Felsenberg, D. (2000). Acute physiological effects of exhaustive whole-body vibration exercise in man. Clinical Physiology, 20(2), 134-142.

Rittweger, J., Mutschelknauss, M., \& Felsenberg, D. (2003). Acute changes in neuromuscular excitability after exhaustive whole body vibration exercise as compared to exhaustion by squatting exercise. Clinical Physiology and Functional Imaging, 23(2), 81-86.

Roelants, M., Delecluse, C., \& Verschueren, S.M. (2004). Whole-body-vibration training increases knee-extension strength and speed of movement in older women. Journal of the American Geriatrics Society, 52(6), 901-908. 
Roelants, M., Verschueren, S.M., Delecluse, C., Levin, O., \& Stijnen, V. (2006). Wholebody-vibration-induced increase in leg muscle activity during different squat exercises. Journal of Strength and Conditioning Research, 20(1), 124-129.

Ronnestad, B.R. (2004). Comparing the performance-enhancing effects of squats on a vibration platform with conventional squats in recreationally resistance-trained men. Journal of Strength and Conditioning Research, 18(4), 839-845.

Runge, M., Rehfeld, G., \& Resnicek, E. (2000). Balance training and exercise in geriatric patients. Journal of Musculoskeletal and Neuron Interaction, 1, 61-65.

Russo, C.R., Lauretani, F., Bandinelli, S., Bartali, B., Cavazzini, C., Guralnik, J.M., et al. (2003). High-frequency vibration training increases muscle power in postmenopausal women. Archives of Physical Medicine and Rehabilitation, 84(12), 1854-1857.

Schlumberger, A., Salin, D., \& Schmidtbleicher, D. (2001). Strength training with superimposed vibrations. Sportverletz Sportschaden, 15(1), 1-7.

Schuhfried, O., Mittermaier, C., Jovanovic, T., Pieber, K., \& Paternostro-Sluga, T. (2005). Effects of whole-body vibration in patients with multiple sclerosis: A pilot study. Clinical Rehabilitation, 19(8), 834-842.

Seidel, H., \& Heide, R. (1986). Long-term effects of whole-body vibration: A critical survey of the literature. International Archives of Occupational and Environmental Health, 58(1), 1-26.

Simoneau, E., Martin, A., \& Van Hoecke, J. (2005). Muscular performances at the ankle joint in young and elderly men. Journals of Gerontology. Series A, Biological Sciences and Medical Sciences, 60(4), 439-447.

Suzuki, T., Bean, J.F., \& Fielding, R.A. (2001). Muscle power of the ankle flexors predicts functional performance in community-dwelling older women. Journal of the American Geriatrics Society, 49(9), 1161-1167.

Taaffe, D.R., \& Marcus, R. (2000). Musculoskeletal health and the older adult. Journal of Rehabilitation Research and Development, 37(2), 245-254.

Thelen, D.G., Schultz, A.B., Alexander, N.B., \& Ashton-Miller, J.A. (1996). Effects of age on rapid ankle torque development. Journals of Gerontology. Series A, Biological Sciences and Medical Sciences, 51(5), M226-M232.

Tinetti, M.E., Speechley, M., \& Ginter, S.F. (1988). Risk factors for falls among elderly persons living in the community. New England Journal of Medicine, 319(26), 17011707.

Torvinen, S., Kannu, P., Sievanen, H., Jarvinen, T.A., Pasanen, M., Kontulainen, S., et al. (2002a). Effect of a vibration exposure on muscular performance and body balance. Randomized cross-over study. Clinical Physiology and Functional Imaging, 22(2), 145-152.

Torvinen, S., Kannus, P., Sievanen, H., Jarvinen, T.A., Pasanen, M., Kontulainen, S., et al. (2002b). Effect of four-month vertical whole body vibration on performance and balance. Medicine and Science in Sports and Exercise, 34(9), 1523-1528.

Verschueren, S.M., Roelants, M., Delecluse, C., Swinnen, S., Vanderschueren, D., \& Boonen, S. (2004). Effect of 6-month whole body vibration training on hip density, muscle strength, and postural control in postmenopausal women: A randomized controlled pilot study. Journal of Bone Mineral Research, 19(3), 352-359.

Vincent, W.J. (2005). Statistics in kinesiology (3rd ed.). Champaign, IL: Human Kinetics. 